\title{
Numerical investigation of space charge effects on the positions of beamlets for transversely split beams
}

\author{
S. Machida and C. Prior \\ ISIS, STFC Rutherford Appleton Laboratory, \\ Harwell Campus, Didcot, Oxfordshire OX11 OQX, United Kingdom \\ S. Gilardoni, M. Giovannozzi, and A. Huschauer \\ Beams Department, CERN, 1211 Geneva 23, Switzerland \\ S. Hirlander \\ Beams Department, CERN, 1211 Geneva 23, Switzerland \\ and Vienna University of Technology, Karlsplatz 13, 1040 Vienna, Austria \\ (Received 3 July 2017; published 15 December 2017)
}

\begin{abstract}
The use of transversely split beams was proposed some years ago as a means to perform extraction from a circular particle accelerator over multiple turns. In the course of studies carried out to increase understanding of the beam behavior, space charge effects have been probed. The experimental results showed a dependence of the beamlets' positions on the total beam intensity. In this paper detailed numerical simulations studies are reported, which clearly indicate that the observed behavior is due to indirect space charge effects. The analysis includes configurations, which have not yet been experimentally probed, in order to better understand the complex interplay between nonlinear single-particle and intensity-dependent effects.
\end{abstract}

DOI: 10.1103/PhysRevAccelBeams.20.121001

\section{INTRODUCTION}

Efforts to find a suitable replacement for the continuous transfer (CT) extraction mode [1,2], which has been the technique used to transfer the $14 \mathrm{GeV} / c$ proton beams from the CERN Proton Synchrotron (PS) to the Super Proton Synchrotron (SPS) for the fixed-target physics programme, converged on the proposal of a novel method of beam manipulation named multiturn extraction (MTE) [3]. This technique is based on transverse beam splitting induced by crossing a stable resonance in the horizontal phase space and solves the issue related to the unavoidable beam losses of the CT extraction [4]. In the case of MTE, the stable fourth-order resonance is used, which generates four beamlets around a central core in phase space. In general, any resonance (stable or unstable) can be considered to design a multi-turn extraction scheme [5]. Furthermore, MTE provides an improved betatron matching of the PS beam at injection in the SPS with respect to CT [6,7].

In the course of experimental studies aimed at preparing the operation of MTE at the PS [8,9] (see also [10-12] for a summary of the MTE performance since it became

\footnotetext{
"massimo.giovannozzi@cern.ch
}

Published by the American Physical Society under the terms of the Creative Commons Attribution 4.0 International license. Further distribution of this work must maintain attribution to the author(s) and the published article's title, journal citation, and DOI. operational in the second half of 2015), the impact of space charge on the beam characteristics was considered and a measurement campaign was carried out, whose results are reported in [13]. The observations can be summarized as follows (see Fig. 1): the final position of the beamlets is a linear function of the total beam intensity, while the rms beam size of the four beamlets and of the beam core do not show any measurable change with total beam intensity. Therefore, the observations can be explained in terms of a positive shift of the linear tune only. The exact origin of the observed displacement of the beamlets' positions is the subject of this paper, in which the contribution from the interaction between the beamlets themselves and that between the beamlets and the boundaries (electric and magnetic) are studied and evaluated by means of numerical simulations.

The studies presented in this paper are not considering the full splitting process as the measurement results indicated that the intensity-dependent effects were affecting solely the beamlets' positions. Hence, the magnetic configuration corresponding to the end of the splitting process is assumed as baseline and the numerical study focuses on the dependence of the fixed points at the centre of the stable islands when a nonzero charge distribution is located inside the islands.

The analysis of intensity-dependent effects for transversely split beams represents an interesting and novel domain, which, by itself, is already an excellent justification for carrying out detailed investigations. Moreover, the 

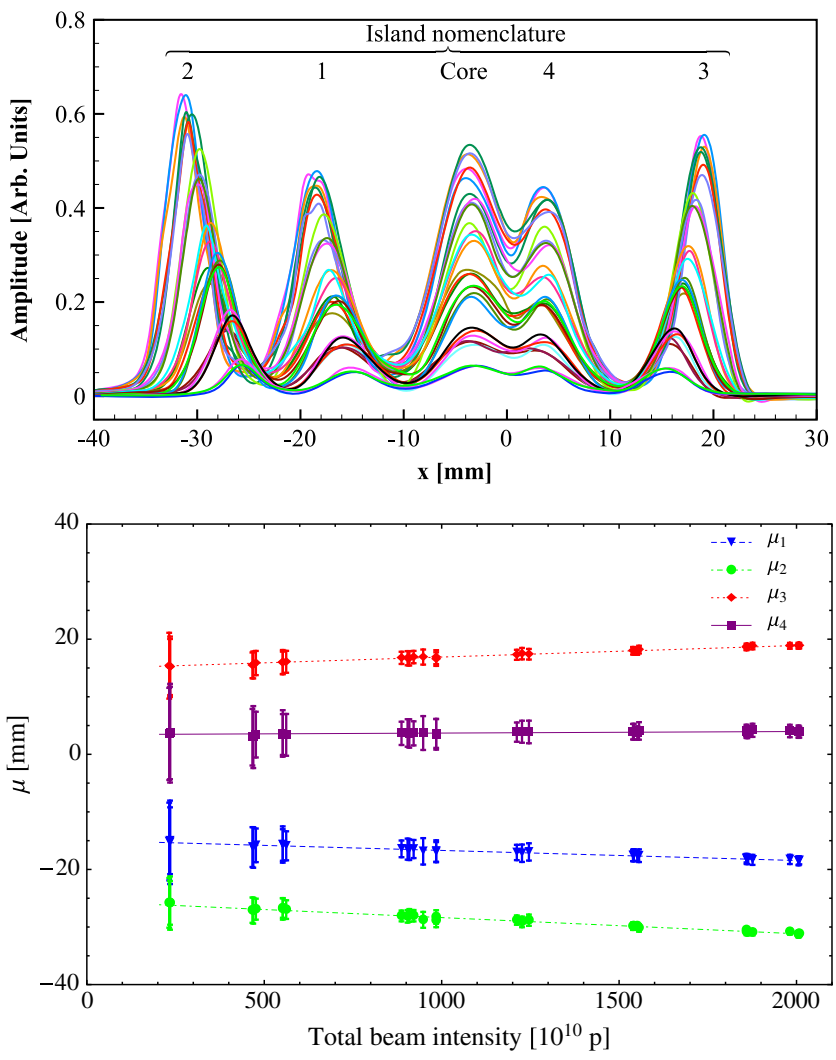

FIG. 1. Summary of the experimental results of intensitydependent effects with split beams from [13]. Upper: horizontal beam profiles measured with a wire scanner at the end of the splitting process with different total beam intensities. Lower: position of the peaks of the beamlets after splitting as a function of the total beam intensity.

possibility of producing high-intensity beams for the proposed future SPS fixed-target facility for the Search for Hidden Particles (SHiP) [14] provides an additional motivation for these studies.

Split beams are possible only thanks to intrinsically nonlinear motion. The generation of stable islands, which are a necessity for split beams, requires nonlinear detuning with amplitude and resonance excitation (see, e.g., [15] for a treatment of nonlinear betatronic motion). The addition of intensity effects generates a peculiar regime whose properties are not well known to date.

In recent years, the interplay between nonlinear effects generated by external fields and those produced by selffields and the interaction with the boundaries has been considered by several authors in studies addressing numerical [16-18], experimental [19-22], and theoretical [23,24] aspects. It is worth stressing that the challenge arises whenever one considers the combined effect of relatively weak external sources of nonlinearities, leading to a sizeable impact on the long-term beam dynamics, and space charge. In fact, this combination requires long-term numerical simulations to shed light on the features of the beam dynamics. However, this is a challenge even in the case of single-particle nonlinear effects, for which a number of numerical tools have been developed for efficient analysis of long-term effects over a relatively short number of turns, with CPU-time at reasonable levels $[25,26]$. The inclusion of intensity-dependent effects complicates the task of simulating the dynamics, both in terms of CPU-time and simulation accuracy. Indeed, a choice for an appropriate paradigm or model to describe the space charge effects has to be made. To describe the evolution of the beam distribution, two different approaches are in general pursued: (i) a relatively fast, albeit not accurate, nonself-consistent (or frozen) treatment, or (ii) a much slower self-consistent approach, which is potentially affected by numerical noise (see, e.g., [27] and references therein). In this respect, the specific problem dealt with in this paper, namely the dependence of the beamlets' positions on beam intensity, considerably simplifies the computational and modelling tasks, shifting the challenges from the computational aspects to the analysis of the interaction of multiple stable beams inside the same boundaries.

The plan of the paper is the following: the detail of the magnetic lattice used in the numerical simulations is discussed in Sec. II. The space charge model used to evaluate the beamlets' positions is presented and discussed in depth in Sec. III and the results of the numerical simulations are discussed in Sec. IV. Finally, conclusions are drawn in Sec. V. The detailed computations of the electric and magnetic fields for specific configurations of the boundary conditions are reported in the Appendices.

\section{MODELING OF PS LATTICE}

The PS lattice (see also [28,29] for more detail) consists of ten superperiods, each made of ten combined function dipole magnets $4.4 \mathrm{~m}$ long, interlaced with eight $1.6 \mathrm{~m}$ and two $3.0 \mathrm{~m}$ long straight sections. Every magnet is composed of two half-units with gradients of opposite sign, separated by a central junction. The latest magnetic measurements using Hall probes [29] showed that stray fields at the magnet ends introduce an additional quadrupolar component, and in the gap between the two halfunits a non-negligible sextupolar component was observed.

The fine adjustment of tunes and chromaticities is performed by means of extra coils mounted on the pole faces of the main dipoles (pole-face-windings-PFWsand figure-of-eight loop-F8L). Up to 2007 these devices were controlled by three independent currents, sufficient to control the two tunes and the horizontal chromaticity. In 2008 an upgrade was implemented and successfully commissioned, allowing independent control of five physical parameters (such as tunes, chromaticities plus one additional physical parameter) by means of five new separate circuits [30]. A sketch of the PS main magnet is shown in the upper part of Fig. 2, while the schematic view of the five circuits to control tunes and chromaticities is shown in the lower part. 

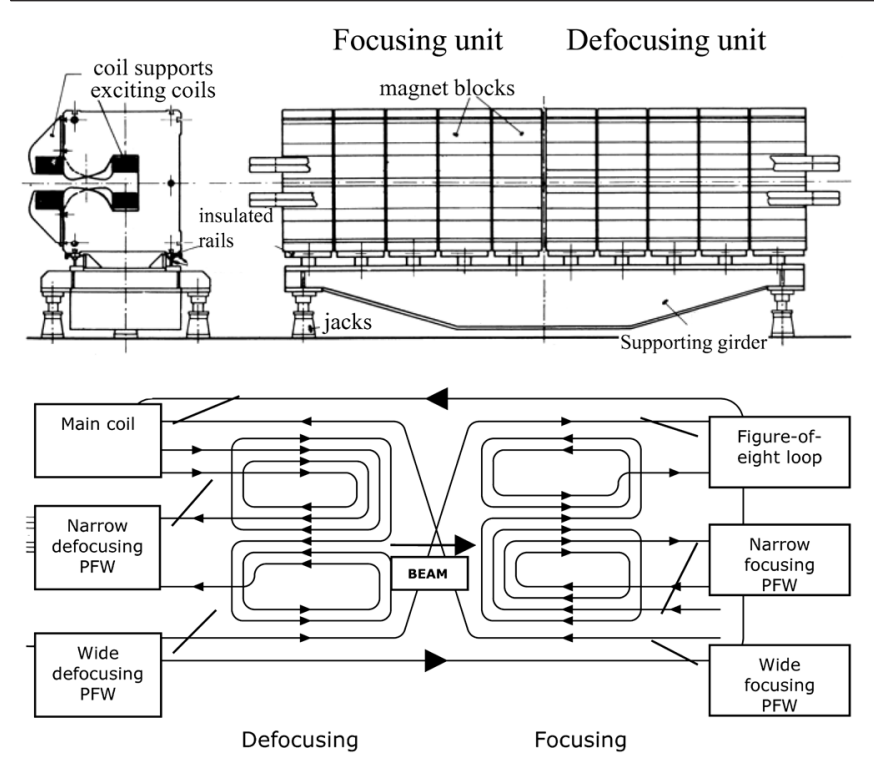

FIG. 2. Sketch of the PS main magnet (upper) and of the five circuits used to control tunes and chromaticities (lower), from [30].

In the PS model used for numerical simulations, the dipole and quadrupole fields generated by the main combined function magnets are fixed by the beam momentum. The additional quadrupolar components induced by stray fields and the PFWs are modeled by thin lenses placed at the beginning and end of each half-unit. At the same locations, sextupolar and higher-order fields generated by the PFWs and by the inter-magnet gap are represented by nonlinear thin lenses. The thin lenses so introduced are eventually grouped into two families, for the focusing and defocusing half-units, respectively.

The effective PS lattice is computed by measuring the transverse tunes as a function of the momentum offset, starting from a given set of horizontal and vertical tunes and chromaticities and beam energy. This beam-based technique has been implemented in the PS since 2002 [31]. A polynomial fit of the measured tune functions is used to extract numerical information on the different magnetic multipoles. The integrated strengths of the thin-lens elements, $K_{n}=1 /(B \rho)\left(\partial^{n} B_{y} / \partial x^{n}\right)$, where $B \rho$ is the magnetic rigidity, are computed to match the measured curves. This procedure is applied order by order up to the octupolar components, i.e., the quadrupolar components are used to reproduce the constant term in the polynomial, the sextupolar components the linear term and so on. The two families (in the defocusing and focusing half-unit of the PS main magnet) of thin-lens elements are used to match the tune curves in the horizontal and vertical planes.

To perform the beam manipulations required for MTE, several families of magnetic elements are used, whose locations are shown in Fig. 3 together with the position of the devices used to measure the transverse beam distributions.

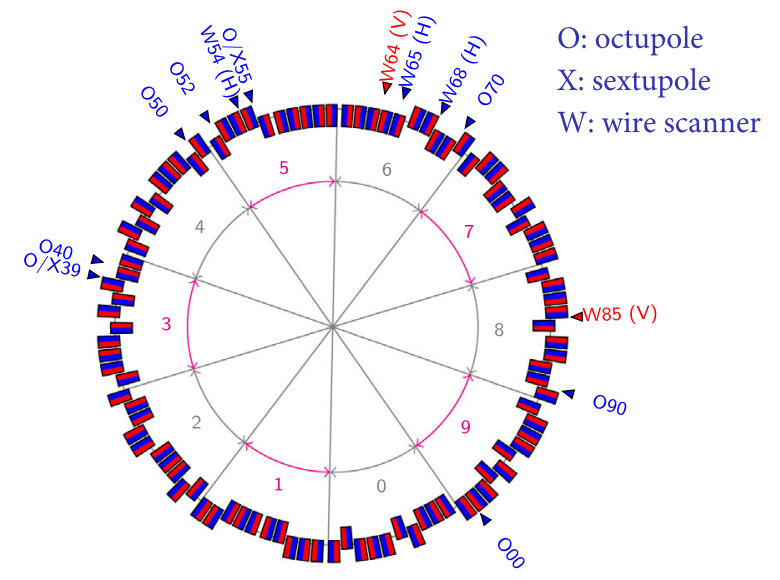

FIG. 3. Layout of the PS ring. The main dipoles are shown (blue and red shapes), including their focusing and defocusing half-units and the way they are installed, i.e., with the yoke inside or outside the circumference. The key elements to perform beam splitting are also shown, the number representing the name of the straight section in which they are installed, and the letter the magnetic type. The wire scanners are indicated together with the plane of measurement.

The control of beam splitting is achieved by means of sextupole and octupole magnets that vary the detuning with amplitude, which is described by the coefficients $h_{2,0}, h_{0,2}, h_{1,1}$ representing the effect of nonlinear motion in the horizontal and vertical plane, as well as the nonlinear coupling between them [15]. Using the following development:

$Q_{x}\left(J_{x}, J_{y}, \delta\right)=Q_{x}+2 h_{2,0} J_{x}+h_{1,1} J_{y}+Q_{x}^{\prime} \delta+\frac{1}{2} Q_{x}^{\prime \prime} \delta^{2}+\cdots$
$Q_{y}\left(J_{x}, J_{y}, \delta\right)=Q_{y}+h_{1,1} J_{x}+2 h_{0,2} J_{y}+Q_{y}^{\prime} \delta+\frac{1}{2} Q_{y}^{\prime \prime} \delta^{2}+\ldots$

where $\delta$ and $J_{x}, J_{y}$ are the relative momentum offset and the actions in the horizontal and vertical planes, respectively, and $Q_{x, y}^{(n)}$ are the $n$th order chromaticities. For sextupoles, the coefficients appearing in Eqs. (1) are given by

$$
\begin{aligned}
& h_{2,0} \propto \sum_{w_{1}, w_{2}} K_{2, w_{1}} K_{2, w_{2}} \lambda_{2, w_{1}, w_{2}} \\
& h_{1,1} \propto \sum_{w_{1}, w_{2}} K_{2, w_{1}} K_{2, w_{2}} \lambda_{1, w_{1}, w_{2}} \\
& h_{0,2} \propto \sum_{w_{1}, w_{2}} K_{2, w_{1}} K_{2, w_{2}} \lambda_{0, w_{1}, w_{2}},
\end{aligned}
$$

where $K_{2}=L /(B \rho)\left(\partial^{2} B_{y} / \partial x^{2}\right), L$ being the length of the magnet and $B_{y}$ the vertical component of the magnetic field. The functions $\lambda_{i, w_{1}, w_{2}}$ with $i=0,1,2$ are complex functions of the beta-functions at the locations $w_{1}, w_{2}$ of the 
sextupoles and of the phase advance between these locations. In the case of octupoles one finds

$$
\begin{aligned}
& h_{2,0} \propto \sum_{w} K_{3, w} \beta_{x, w}^{2} \\
& h_{1,1} \propto \sum_{w} K_{3, w} \beta_{x, w} \beta_{y, w} \\
& h_{0,2} \propto \sum_{w} K_{3, w} \beta_{y, w}^{2},
\end{aligned}
$$

where $K_{3}=L /(B \rho)\left(\partial^{3} B_{y} / \partial x^{3}\right)$.

The configuration used for the numerical simulations corresponds to the one of [13]. It is worth noting that the value of the detuning with amplitude in the horizontal plane, i.e., the term $h_{2,0}$, is negative, which implies that stable islands are present whenever the horizontal tune satisfies $Q_{x}>6.25$. An additional configuration has been probed in numerical simulations, namely with a positive value of $h_{2,0}$.

\section{MODELING OF INTENSITY-DEPENDENT EFFECTS}

The numerical simulations presented in this paper have been carried out using the space charge simulation code SIMPSONS [32] with a number of additions needed for this study. For instance, the algorithm for finding the closed orbit after $N$ turns, where $N$ is the order of nonlinear resonance, has been implemented, in addition to the usual closed orbit search over a single turn. Summation of space charge kicks from multiple bunches, which represent the transversely split beamlets, with the appropriate boundary conditions is another feature that was not available in the original version of the code.

The dependence of the positions of the beamlets on the total beam intensity resembles a single-particle problem, namely the computation of fixed points for a nonlinear system. Some models have been proposed [23,24], inspired by the single-particle approach, describing the dynamics of a test particle under the influence of external fields generated by the lattice magnets and internal fields generated by a beam with a frozen charge distribution located at the center of phase space (the equilibrium orbit of the system). The scenario considered in our studies corresponds to the analysis of the incoherent motion of the test particle.

In our problem, the equilibrium orbit for the system of multiple beamlets under the influence of the various forces acting on them has to be determined. To our best knowledge, no analytical model has ever been proposed to describe this configuration and only numerical computations can provide the correct answer.

The algorithm relies on an iterative process: $\delta$-like charge distributions are initially located on the fixed points of the system governed by the external fields only, i.e., assuming zero-beam intensity. Then, this configuration is tracked for a few turns including also the self-fields. As the initial positions of the charge distributions do not correspond to the equilibrium configuration of the complete system, which includes also internal fields, the beamlets oscillate around the true equilibrium. Therefore, the centers of these oscillations can provide a better approximation of the equilibrium positions and this closes the iterative process, as these centers provide a new initial condition for another iteration. The process ends when the difference between the initial condition and the final equilibrium orbit reaches a threshold value. An example of the iterative procedure is shown in Fig. 4, where the difference between the initial guess of the position of one beamlet and its true positions is reported as a function of the iteration number of the procedure. Note that the charge distribution located at the center of phase space, unlike the outer beamlets, never oscillates during the iterative process.

In the numerical implementation of the algorithm, the $\delta$-like charge distributions are each replaced by a uniform distribution over a disk of radius $R$, always much smaller than the surface of the stable island for the system with zero beam intensity. A convergence check has been performed, which showed that the numerical results are independent of the value of $R$, at least when $R$ is in the range $0.2-5 \mathrm{~mm}$. In the following computations, $R=1 \mathrm{~mm}$ is used.

Interactions between the charge distributions and the boundaries are computed at discrete points around the ring circumference with a spacing between kicks of $\Delta \mathrm{cm}$. The approach used is that of a frozen model, i.e., the charge distributions are kept unchanged during the steps around the ring circumference. Also in this case the stability of numerical results as a function of $\Delta$ has been checked and, for the numerical simulations shown in the following, $\Delta=33 \mathrm{~cm}$ is used.

The effect of the boundaries has been taken into account by solving the Poisson equation for the potential. In the

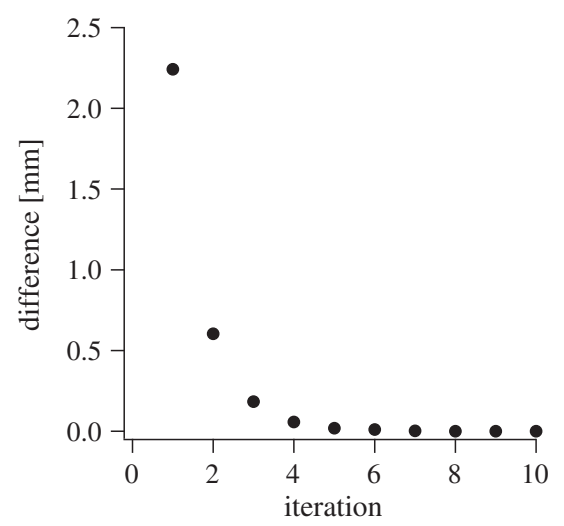

FIG. 4. Iterative process to determine the self consistent position of the beamlets. The vertical axis describes the distance of a beamlet's position from the closed orbit found after particle tracking including self-fields. 
case of electric boundary conditions, two shapes have been considered, namely perfectly conducting horizontal parallel plates or a perfectly conducting rectangle. Note that the standard PS vacuum pipe is elliptical in shape, with dimensions $140 \times 70 \mathrm{~mm}^{2}$. The beam is split in the horizontal phase space, but the vertical beam distribution is unaffected and remains a standard Gaussian. Therefore, the presence of vertical boundaries has been considered a feature to be probed by the numerical simulations, while the variation of the vertical beam pipe dimension as a function of the horizontal position has been considered less important. Based on these arguments two configurations have been considered in our studies, namely parallel and rectangular perfectly conducting electric boundaries.

The expressions for the electric field are derived in Appendix A and B. The vertical dimension of the standard vacuum chamber has been assumed for both configurations. Similarly, the horizontal dimension of the standard vacuum chamber has been assumed for the rectangular boundary condition. The magnetic boundary conditions, given by the pole faces of the PS combined function main magnets, have been approximated by parallel plates with half gap of $50 \mathrm{~mm}$. Such a value corresponds to distance between the median plane and the magnet pole face at the location of the reference equilibrium orbit. In this case, the closed form of the magnetic potential is given in Appendix A.

\section{RESULTS OF NUMERICAL SIMULATIONS}

The models described in the previous section have been used to study the behavior of the beamlets' positions as a function of several parameters describing the system under study. In addition, the general phase space topology has been considered, together with some special configurations.

\section{A. Phase space topology}

The horizontal phase space portraits of the PS lattice are shown in Fig. 5 for negative (upper left) and positive (lower left) values of $h_{2,0}$ and without space charge effects.

This special configuration features opposite-sign octupolar components with respect to that used in the experiments. According to Eqs. (3) a change of sign of $K_{3}$ generates a change of sign of all octupolar contributions to the quantities $h_{i, j}$. This is, however, not the case for the sextupolar contributions of Eqs. (2), which remain unchanged for a change of sign of $K_{2}$. The overall result is that a sign reversal of the octupolar components in the PS lattice model does not exactly reverse the sign of the $h_{i, j}$. Hence, to obtain islands that are located approximately at the same amplitude in phase space (see Fig. 5) the required tune value is not exactly symmetrical with respect to 6.25 . This effect is clearly seen in Fig. 6, where the phase space
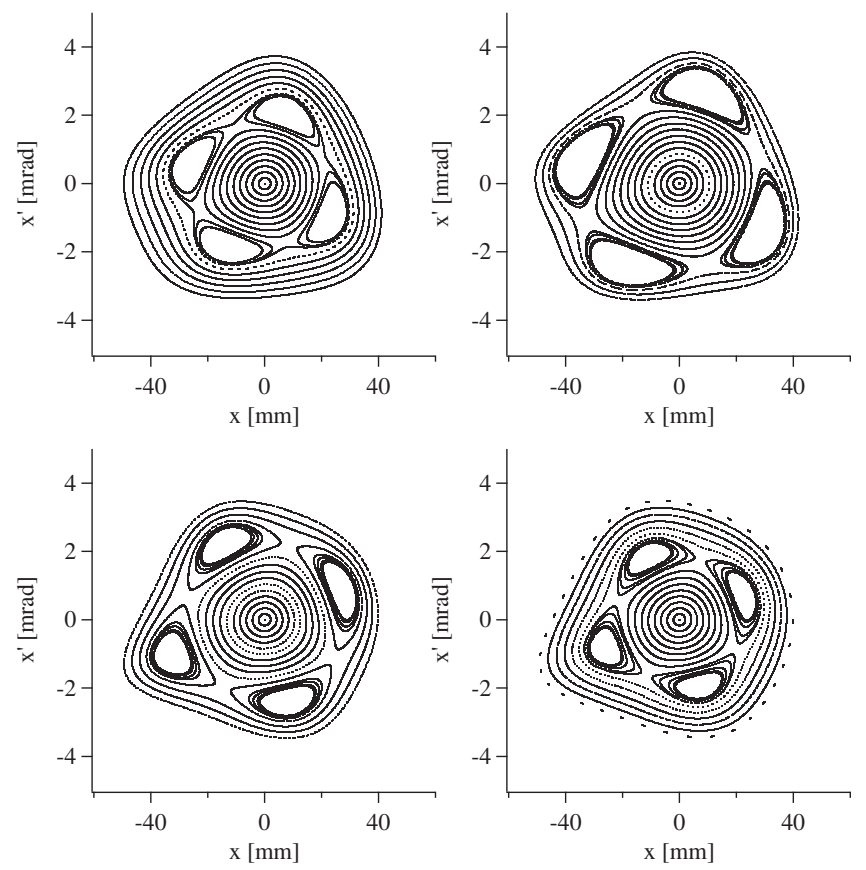

FIG. 5. Upper left: Phase space portrait corresponding to the PS configuration used in the experimental studies without space charge effects, with $h_{2,0}<0$ and $Q_{x}=6.255$. Upper right: Phase space portrait corresponding to the configuration of upper left plot including five equally charged beamlets for a total intensity of $3.28 \times 10^{13}$ p. Lower left: Phase space portrait corresponding to a special PS configuration used only for the numerical simulations without space charge effects, with $h_{2,0}>0$ and $Q_{x}=6.245$. Lower right: Phase space portrait corresponding to the configuration of the lower left plot including five equally charged beamlets for a total intensity of $1.31 \times 10^{13} \mathrm{p}$. Parallel plates electric boundary conditions are included. The beam momentum is $14 \mathrm{GeV} / c$.

portraits applying the two different tune values, below and above the resonance value, are overlaid.

While the stable fixed points are approximately at the same distance from the origin, the phase differs between the two configurations. Moreover, the overall shape of closed curves beyond the chain of stable islands is also not exactly the same for the two cases.

This can also be seen in Fig. 7 where the position of one of the fixed points is shown as a function of the linear tune for the two cases corresponding to opposite sign of the value of $h_{2,0}$.

\section{B. Fixed point position}

The focus of our study is the behavior of the beamlets' positions as a function of several parameters describing the system under study. It is worth mentioning that in the following the beamlets' positions are given at the PS section 64 (see Fig. 3), which corresponds to the location of one of the wire scanners used in the experimental campaign [13]. Moreover, among the four possible, only 


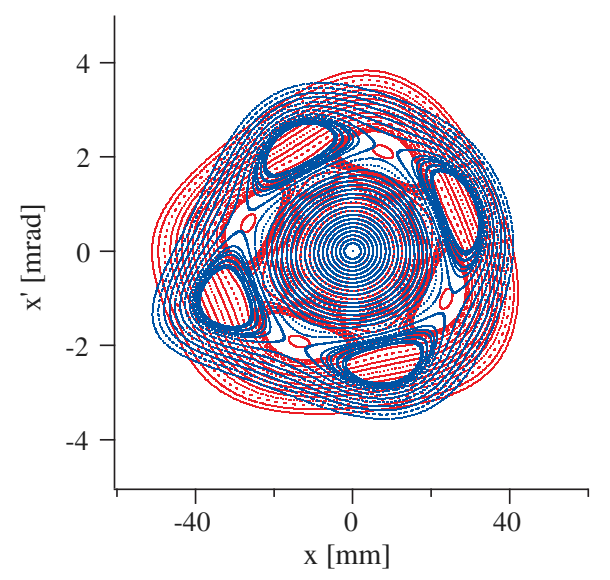

FIG. 6. Superposition of the transverse phase space corresponding to a horizontal tune of 6.255 and $h_{2,0}<0$ (red) and a tune of 6.245 and $h_{2,0}>0$ (blue). In both cases no intensity-dependent effects are taken into account.

one beamlet has been selected to show the dependence on the system's parameters, namely that featuring $x>0$ and $x^{\prime}>0$.

As a global test, the two configurations shown in the left column of Fig. 5 have been used to compute phase space portraits in the presence of charges located at the beamlets' positions. The results of the numerical simulations are shown in the right column of Fig. 5. Differences induced by the intensity-dependent effects are clearly visible in terms of beamlets' positions, islands' sizes, and the presence of chains of small islands beyond those of the fourth-order resonance.

Figure 8 shows one of the main results of the numerical simulations, namely the dependence of the beamlet's position on the total beam intensity. For the sake of comparison, the results for the two configurations with $h_{2,0}>0$ (upper plot) and $h_{2,0}<0$ (lower plot) are shown.

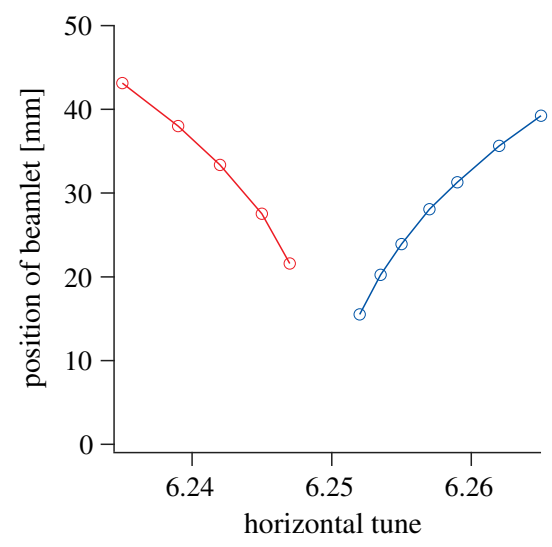

FIG. 7. Evolution of the position of one of the fixed points as a function of the horizontal tune for the two cases with negative (blue) and positive (red) value of $h_{2,0}$. In both cases no intensitydependent effects are taken into account.
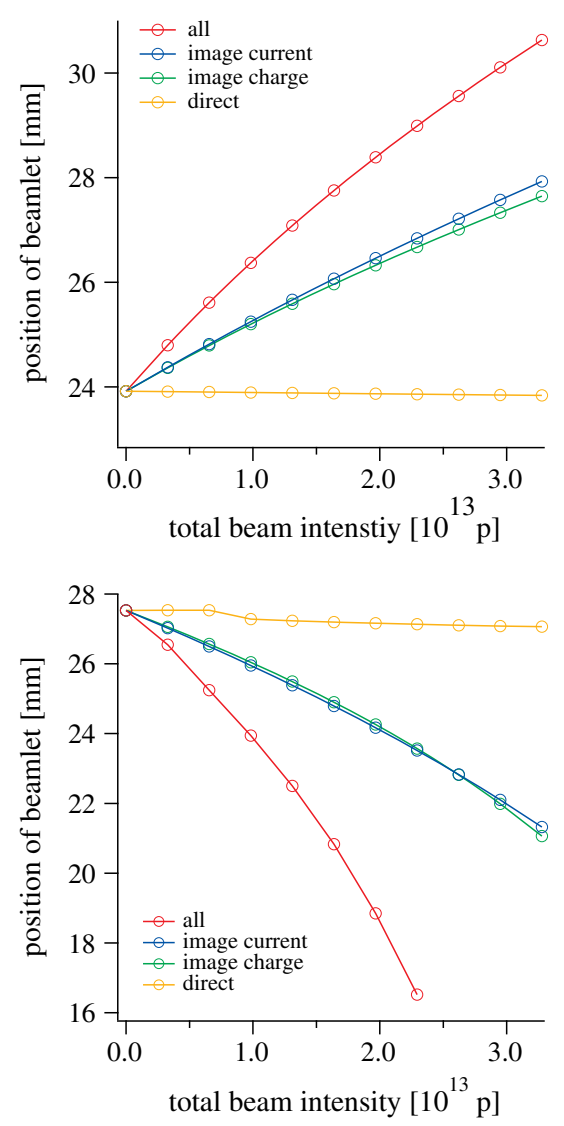

FIG. 8. Beamlet position as a function of the total beam intensity. The cases corresponding to $h_{2,0}<0, Q_{x}=6.255$ and $h_{2,0}>0, Q_{x}=6.245$ are shown in the upper and lower plot, respectively. The contribution of the various sources of intensitydependent position shifts is indicated by the various curves. Parallel plate electric boundary conditions are included. The beam momentum is $14 \mathrm{GeV} / c$.

Moreover, the impact of the various intensity-dependent effects, namely direct space charge, electric images, and magnetic images, is evaluated separately as well as combined to provide the overall effect.

Direct space charge has the weakest effect on the beamlet's position and, as expected, the sign is constant, i.e., independent of the sign of $h_{2,0}$. For the boundary types considered in these simulations the electric and magnetic effects are very similar in magnitude and their sign does depend on that of $h_{2,0}$. This is in qualitative agreement with the experimental observations [13], where the configuration featured $h_{2,0}<0$ and the beamlets' positions were increasing with increasing total beam intensity.

It is also worth noting that the variation of the beamlet's position with total beam intensity also depends on the value of $Q_{x}$. This aspect has been studied in more detail and the results of numerical simulations are shown in Fig. 9, where the variation of the beamlet's position vs total beam intensity is shown for several values of $Q_{x}$. The dependence on $Q_{x}$ is clearly visible. 

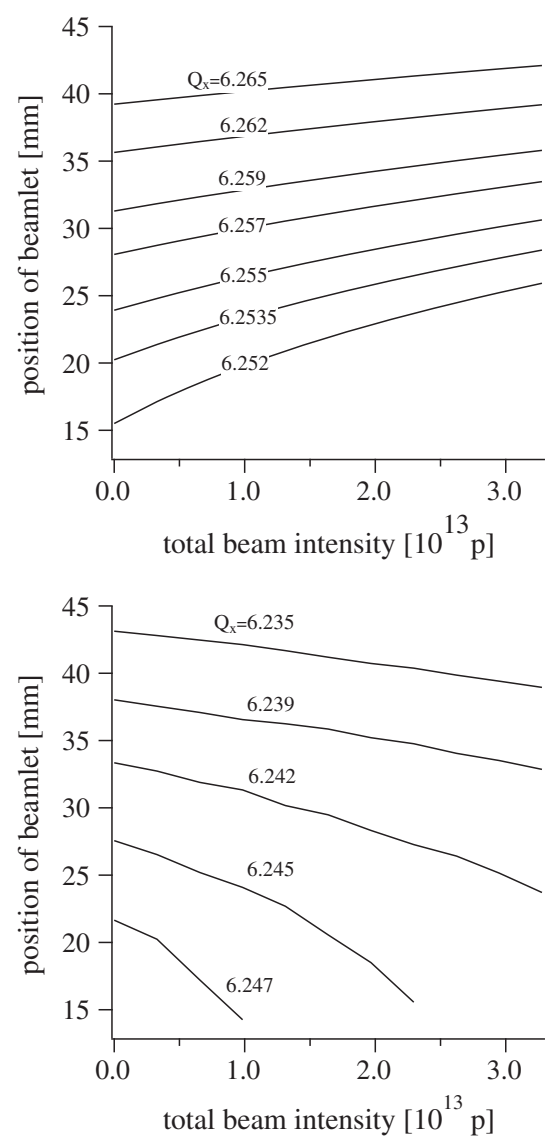

FIG. 9. Dependence of a beamlet's position on the total intensity. Several values of the horizontal tune, generating different beam positions for zero intensity, are probed. The cases corresponding to $h_{2,0}<0$ and $h_{2,0}>0$ are shown in the upper and lower plots, respectively. For $Q_{x}=6.247$ and 6.245, the fixed points disappear for the largest intensity reported in the plot. Parallel plates electric boundary conditions are included. The beam momentum is $14 \mathrm{GeV} / c$.

Furthermore, the impact of different types of electric boundary conditions, namely parallel plates and rectangular, has been tested with a series of dedicated numerical simulations and the results are shown in Fig. 10 for both signs of $h_{2,0}$.

The differences are small, which can be understood by the fact that the beamlets are close to the vertical electric boundary only over a small fraction of the ring circumference. Therefore, the existence of vertical boundaries can only have a minor impact on the beamlets' dynamics.

The last parameter that has been considered in these numerical studies is the beam momentum $p$, and the dependence of the beamlet's position on $p$ is shown in Fig. 11, where the different contributing effects are reported separately.

The direct space charge tends to move the beamlet in a direction opposite to the effect seen in the interaction with the boundaries. Moreover, while the effect of the direct space charge levels off around $6 \mathrm{GeV} / c$, that of the
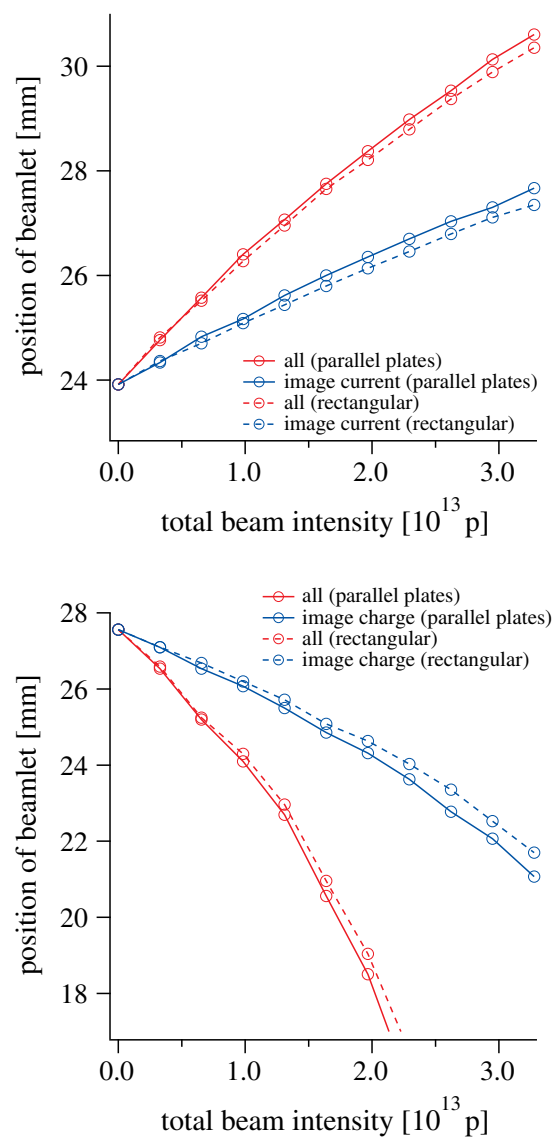

FIG. 10. Beamlet's position as a function of the total beam intensity. The impact of the different electric boundary conditions is small, but visible. The cases corresponding to $h_{2,0}<0$, $Q_{x}=6.255$ and $h_{2,0}>0, Q_{x}=6.245$ are shown in the upper and lower plot, respectively. The contribution of the various sources of intensity-dependent position shifts is indicated by the various curves. The beam momentum is $14 \mathrm{GeV} / c$.

interaction with the boundaries continues to change even at higher values of the beam momentum.

\section{Special configurations}

The total beam intensity is clearly a parameter with strong impact on the beamlets' positions. However, it is also possible to consider special configurations in which the intensity sharing between the outer beamlets and the central one is not even. This generates another class of configurations that can be classified by means of the socalled MTE efficiency [10-12] defined as

$$
\text { MTE efficiency }=\eta_{\mathrm{MTE}}=\frac{\left\langle I_{\text {Islands }}\right\rangle}{I_{\text {Total }}} \text {. }
$$

For MTE, the nominal value is $\eta_{\mathrm{MTE}}=0.2$, corresponding to an equal sharing of the total beam intensity between the five beamlets: this is the value assumed for the numerical simulations presented so far. Lower values indicate that the 


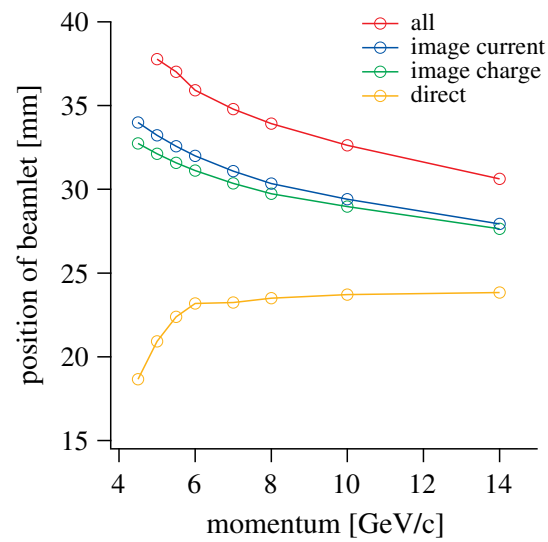

FIG. 11. Beamlet's position as a function of the beam momentum (the total beam intensity is $3.28 \times 10^{13} \mathrm{p}$ ) for $h_{2,0}<0$ and $Q_{x}=6.255$ and parallel plates electric boundary conditions.

central beamlet is more populated than the outer ones; the limiting case $\eta_{\mathrm{MTE}}=0$ represents an unsplit beam. The opposite occurs for $\eta_{\mathrm{MTE}}>0.2$, where the limiting case $\eta_{\mathrm{MTE}}=0.25$ represents a beam without the central beamlet. In Fig. 12 the beamlet's position is shown as a function of $\eta_{\mathrm{MTE}}$.

A linear behavior is clearly visible. This can be understood considering that each beamlet has an intensity equal to $I_{\text {Total }} \eta_{\text {MTE }}$ while the core has an intensity $I_{\text {Total }}\left(1-4 \eta_{\text {MTE }}\right)$. The effects due to the interactions with the boundaries are linear in the intensity of each beamlet and hence linear in $\eta_{\mathrm{MTE}}$, while the core does not contribute. Whenever direct space-charge effects have to be taken into account, the pairwise interaction between beamlets and between beamlets and core should also be included. In this case the beamlets provide a contribution that scales with $I_{\text {Total }}^{2} \eta_{\text {MTE }}^{2}$, while a beamlet and the core contribute a term of the form $I_{\text {Total }}^{2} \eta_{\mathrm{MTE}}\left(1-4 \eta_{\mathrm{MTE}}\right)$. In summary, if direct space-charge can be neglected, the

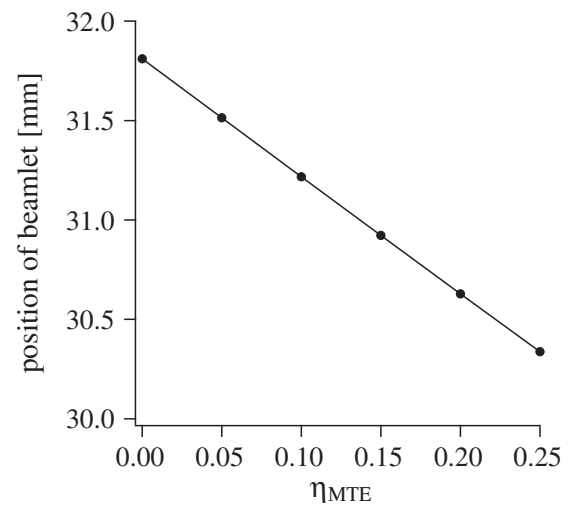

FIG. 12. Beamlet's position as a function of MTE efficiency with $h_{2,0}<0$ and $Q_{x}=6.255$ (the total beam intensity is $3.28 \times 10^{13} \mathrm{p}$ ) and parallel plates electric boundary conditions.

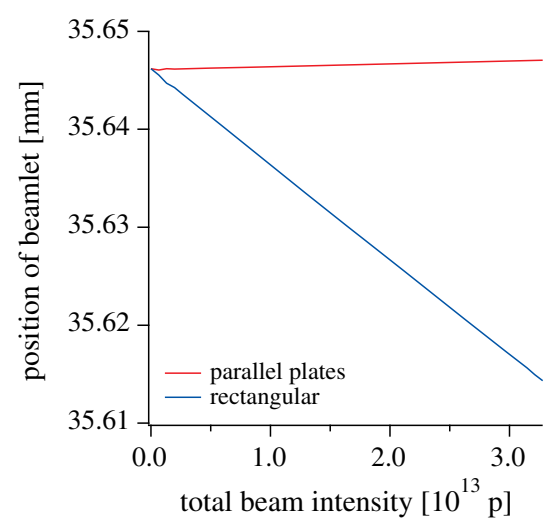

FIG. 13. Beamlet's position for a beam kicked into a stable island. Two different electric boundary conditions have been used. In this case $h_{2,0}<0$ and $Q_{x}=6.262$. The beam momentum is $14 \mathrm{GeV} / c$.

beamlets' positions should scale linearly with $\eta_{\mathrm{MTE}}$, else terms in $\eta_{\mathrm{MTE}}^{2}$ have to be included. It is worth noting that the dependence on $I_{\text {Total }}$ is linear without direct space charge, otherwise it is quadratic.

The last study presented here considers a special configuration that can be achieved when a Gaussian beam is not split, but is simply displaced into a stable island. In this case, the only intensity-dependent effect is due to the interaction of the single beamlet with the boundaries (electric and magnetic). The results of the numerical simulations are shown in Fig. 13, where the beamlet position is plotted as a function of the total intensity for the two types of electric boundary conditions.

In the case of electric boundary conditions given by parallel plates the beamlet position is nearly independent of the total beam intensity. On the other hand, whenever rectangular electric conditions are considered a small nonzero shift of the beamlet's position with intensity is observed.

\section{CONCLUSIONS}

In this paper the dependence of the positions of the beamlets obtained after splitting a beam by means of a stable fourth-order resonance has been analysed as a function of the total beam intensity. This novel topic is considered for the first time, and the study is aimed at clarifying the experimental results obtained at the CERN PS ring during transverse beam splitting by means of resonance crossing.

The numerical analysis presented in this paper covers the detail of the possible sources of beamlets' positions displacement with intensity, namely direct space charge as well as indirect effects (electric and magnetic). The analysis is based on a frozen space-charge model, which is perfectly adequate for the phenomenon under consideration. The dependence of the beamlets' positions under 
the influence of intensity effects has also been examined for different values of the horizontal tune, of the MTE efficiency, and of the beam momentum.

The main conclusion is that a clear interplay has been seen for the first time between nonlinear effects due to external sources, like magnetic elements distributed along the ring, and intensity effects (direct and indirect). A crucial point is that, by changing the sign of the detuning with amplitude generated by nonlinear magnetic elements, it has been possible to change the sign of the variation of the fixed points positions vs intensity. The magnitude of the displacement of the fixed points is both amplitude- and intensity-dependent, thus indicating the close connection between nonlinear and space charge effects. All this indicates that compensation, at least partial, between the magnetic nonlinearities and those generated by the intensitydependent effects might occur.

A direct and quantitative comparison with the experimental results obtained at CERN is not possible, due to the difference in the models used to describe the boundary conditions, namely the vacuum chambers and the pole shapes of the PS main magnets. However, the experimental observations are qualitatively fully confirmed by the analysis presented here. In particular, it is worth stressing that the increased distance of the fixed points from the origin when the intensity is increased has been confirmed, in agreement with the experimental observations. Furthermore, the origin of this behavior has been clearly identified and linked to indirect effects, which represents a step forward in the understanding of the phenomenon under consideration.

While the main focus of these studies is the analysis of the fixed point dependence on intensity whenever five beamlets are present in a ring, special simulations have been performed to understand the behavior when a single beamlet is displaced inside a stable island. The numerical results indicate that a shift of the fixed point may also occur in this case, due to indirect space charge effects. Nevertheless, the effect may be small and, in any case, dependent on the geometry of the electric and magnetic boundary conditions.

\section{APPENDIX A: ELECTRIC AND MAGNETIC FIELDS FOR PARALLEL PLATES CONFIGURATION OF BOUNDARY CONDITIONS}

The electric field generated by a charge distribution with linear density $\lambda$ at a position $\left(x_{0}, 0\right)$ between two perfectly conducting, horizontally oriented, parallel plates located at a vertical position of $\pm h / 2$ can be found, e.g., by using the method of images [33], i.e., by summing up the contributions to the electric field from alternating sign, virtual charges according to

$$
\begin{aligned}
& E_{x}(x, y)=\frac{\lambda}{2 \pi \varepsilon_{0}}\left(x-x_{0}\right) \sum_{n=-\infty}^{+\infty} \frac{(-1)^{n}}{\left(x-x_{0}\right)^{2}+\left(y+2 n \frac{h}{2}\right)^{2}} \\
& E_{y}(x, y)=\frac{\lambda}{2 \pi \varepsilon_{0}} \sum_{n=-\infty}^{+\infty} \frac{(-1)^{n}\left(y+2 n \frac{h}{2}\right)}{\left(x-x_{0}\right)^{2}+\left(y+2 n \frac{h}{2}\right)^{2}}
\end{aligned}
$$

where $\varepsilon_{0}$ is the permittivity of free space. An explicit expression for the two series can be found and the final result reads

$$
\begin{aligned}
& E_{x}(x, y)=-\frac{\lambda}{h \varepsilon_{0}} \frac{\cos \frac{\pi}{h} y \sinh \frac{\pi}{h}\left(x-x_{0}\right)}{\left[\cos \frac{2 \pi}{h} y-\cosh \frac{2 \pi}{h}\left(x-x_{0}\right)\right]} \\
& E_{y}(x, y)=-\frac{\lambda}{h \varepsilon_{0}} \frac{\sin \frac{\pi}{h} y \cosh \frac{\pi}{h}\left(x-x_{0}\right)}{\left[\cos \frac{2 \pi}{h} y-\cosh \frac{2 \pi}{h}\left(x-x_{0}\right)\right]} .
\end{aligned}
$$

An example of the electric potential $\phi=-\operatorname{grad}\left(E_{x}, E_{y}\right)$ is shown in Fig. 14.

Similarly, the magnetic field generated by a current $I$ at a position $\left(x_{0}, 0\right)$ between two ferromagnetic, horizontally oriented, parallel plates located at a vertical position of $\pm g / 2$ can be found by placing virtual currents at appropriate positions. The expression for the magnetic field has the form

$$
\begin{aligned}
& B_{x}(x, y)=-\frac{I \mu_{0}}{2 \pi} \sum_{n=-\infty}^{+\infty} \frac{y+2 n \frac{g}{2}}{\left(x-x_{0}\right)^{2}+\left(y+2 n \frac{g}{2}\right)^{2}} \\
& B_{y}(x, y)=\frac{I \mu_{0}}{2 \pi}\left(x-x_{0}\right) \sum_{n=-\infty}^{+\infty} \frac{1}{\left(x-x_{0}\right)^{2}+\left(y+2 n \frac{g}{2}\right)^{2}},
\end{aligned}
$$

where $\mu_{0}$ is the permeability of free space. Also in this case and explicit expression for the magnetic field can be derived and it reads

$$
\begin{aligned}
& B_{x}(x, y)=\frac{I \mu_{0}}{2 g} \frac{\sin \frac{2 \pi}{g} y}{\left[\cos \frac{2 \pi}{g} y-\cosh \frac{2 \pi}{g}\left(x-x_{0}\right)\right]} \\
& B_{y}(x, y)=-\frac{I \mu_{0}}{2 g} \frac{\sinh \frac{2 \pi}{g}\left(x-x_{0}\right)}{\left[\cos \frac{2 \pi}{g} y-\cosh \frac{2 \pi}{g}\left(x-x_{0}\right)\right]} .
\end{aligned}
$$

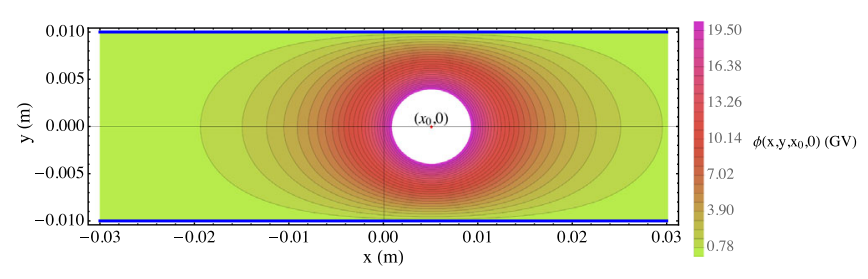

FIG. 14. Contour plot of the electric potential $\phi$ for a unit charge density located in $\left(x_{0}, 0\right)$. The boundary surfaces are shown in blue. 


\section{APPENDIX B: ELECTRIC FIELDS FOR RECTANGULAR BOUNDARY CONDITIONS}

A more realistic case for accelerators, is the electric field generated by a charge distribution with linear density $\lambda$ at a position $\left(x_{0}, 0\right)$ inside a perfectly conducting rectangular shape. Explicit solutions for this case can be found in the literature (see, e.g., Refs. [34,35]).

Assuming that the rectangle is described by parameters $a$ and $b$ representing its half width and height, respectively, then let $K(m)$ denote the complete elliptic integral of first kind, i.e.,

$$
K(m)=\int_{0}^{\pi / 2} \frac{d \theta}{\sqrt{1-m \sin ^{2} \theta}}
$$

where the parameter $m$ is defined implicitly by the following equation

$$
\frac{2 b}{a}=\frac{K\left(1-m^{2}\right)}{K\left(m^{2}\right)} .
$$

Additional functions should be defined to express the solution and they are linked to the elliptic integral of first kind $F(\phi \mid m)$

$$
F(\phi \mid m)=\int_{0}^{\phi} \frac{d \theta}{\sqrt{1-m \sin ^{2} \theta}} .
$$

The inverse of $F(\phi \mid m)$ is called amplitudinis function $\operatorname{am}(\phi \mid m)$

$$
\phi=\int_{0}^{\mathrm{am}(\phi \mid m)} \frac{d \theta}{\sqrt{1-m \sin ^{2} \theta}} .
$$

It is then possible to define trigonometric functions of $\operatorname{am}(t \mid m)$, namely

$$
\begin{aligned}
\sin (\operatorname{am}(t \mid m)) & =\operatorname{sn}(t \mid m) \\
\cos (\operatorname{am}(t \mid m)) & =\operatorname{cn}(t \mid m)
\end{aligned}
$$

and the Jacobi elliptic function $\operatorname{dn}(t \mid m)$ defined as:

$$
\operatorname{dn}(t \mid m)=\sqrt{1-m \operatorname{sn}(t \mid m)^{2}}
$$

The electric field that satisfies the Poisson equation with rectangular boundary conditions can be written, using a complex notation where ${ }^{*}$ denotes complex conjugation, as

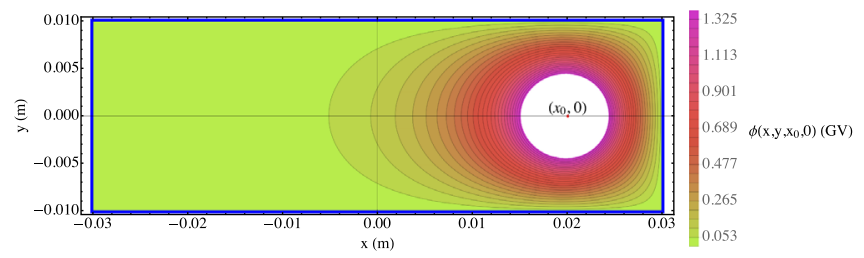

FIG. 15. Contour plot of the electric potential $\phi$ for a unit charge density located in $\left(x_{0}, 0\right)$. The boundary surfaces are shown in blue.

$$
\begin{aligned}
& E_{x}(x+i y)+i E_{y}(x+i y) \\
& =\lambda \frac{K\left(1-m^{2}\right)}{32 \pi b \varepsilon_{0}} \\
& \quad \times\left\{\frac{\mathrm{Cn}(x+i y) \operatorname{Dn}(x+i y)\left[m \mathrm{Sn}^{2}\left(x_{0}\right)-1\right]}{\left[\operatorname{Sn}(x+i y)-\operatorname{Sn}\left(x_{0}\right)\right]\left[m \operatorname{Sn}(x+i y) \operatorname{Sn}\left(x_{0}\right)-1\right]}\right\}^{*}
\end{aligned}
$$

where $\left(x_{0}, 0\right)$ stand for the position of the charge distribution of line density $\lambda$ and the following identities have been used

$$
\begin{aligned}
& \operatorname{Sn}(z)=\operatorname{sn}\left(\frac{K\left(1-m^{2}\right)}{2}(z+i) \mid m^{2}\right) \\
& \operatorname{Cn}(z)=\operatorname{cn}\left(\frac{K\left(1-m^{2}\right)}{2}(z+i) \mid m^{2}\right) \\
& \operatorname{Dn}(z)=\operatorname{dn}\left(\frac{K\left(1-m^{2}\right)}{2}(z+i) \mid m^{2}\right),
\end{aligned}
$$

with $z=x+i y$.

An example of the electric potential $\phi=-\operatorname{grad}\left(E_{x}, E_{y}\right)$ is shown in Fig. 15. The deformation of the level lines of the electric potential due to the vertical boundaries is clearly visible.

[1] C. Bovet, D. Fiander, L. Henny, A. Krusche, and G. Plass, in 1973 Particle Accelerator Conference, San Francisco, CA, USA, 1973, edited by E. J. Lofgren (IEEE, New York, 1973), p. 438.

[2] D. C. Fiander, D. Grier, K.-D. Metzmacher, and P. Pearce, A modulated fast bump for the CPS continuous transfer, IEEE Trans. Nucl. Sci. 24, 1340 (1977).

[3] R. Cappi and M. Giovannozzi, Novel Method for MultiTurn Extraction: Trapping Charged Particles in Islands of Phase Space, Phys. Rev. Lett. 88, 104801 (2002).

[4] J. B. García and S. Gilardoni, Simulation and optimization of beam losses during continuous transfer extraction at the CERN Proton Synchrotron, Phys. Rev. ST Accel. Beams 14, 030101 (2011).

[5] R. Cappi and M. Giovannozzi, Multiturn extraction and injection by means of adiabatic capture in stable islands 
of phase space, Phys. Rev. ST Accel. Beams 7, 024001 (2004).

[6] R. Cappi and M. Giovannozzi, Report No. CERN PS 2002083, 2002.

[7] R. Cappi and M. Giovannozzi, Multiturn extraction: Performance analysis of old and new approaches, Nucl. Instrum. Methods Phys. Res., Sect. A 519, 442 (2004).

[8] S. Gilardoni, M. Giovannozzi, M. Martini, E. Métral, P. Scaramuzzi, R. Steerenberg, and A.-S. Müller, Experimental evidence of adiabatic splitting of charged particle beams using stable islands of transverse phase space, Phys. Rev. ST Accel. Beams 9, 104001 (2006).

[9] A. Franchi, S. Gilardoni, and M. Giovannozzi, Progresses in the studies of adiabatic splitting of charged particle beams by crossing nonlinear resonances, Phys. Rev. ST Accel. Beams 12, 014001 (2009).

[10] J. Borburgh, S. Damjanovic, S. Gilardoni, M. Giovannozzi, C. Hernalsteens, M. Hourican, A. Huschauer, K. Kahle, G. Le Godec, O. Michels, and G. Sterbini, First implementation of transversely split proton beams in the CERN Proton Synchrotron for the fixed-target physics programme, Europhys. Lett. 113, 34001 (2016).

[11] S. Abernethy, A. Akroh, H. Bartosik, A. Blas, T. Bohl, S. Cettour-Cave, K. Cornelis, H. Damerau, S. Gilardoni, M. Giovannozzi, C. Hernalsteens, A. Huschauer, V. Kain, D. Manglunki, G. Métral, B. Mikulec, B. Salvant, J.-L. Sanchez Alvarez, R. Steerenberg, G. Sterbini, and Y. $\mathrm{Wu}$, Operational performance of the CERN injector complex with transversely split beams, Phys. Rev. Accel. Beams 20, 014001 (2017).

[12] A. Huschauer, A. Blas, J. Borburgh, S. Damjanovic, S. Gilardoni, M. Giovannozzi, M. Hourican, K. Kahle, G. Le Godec, O. Michels, G. Sterbini, and C. Hernalsteens, Transverse beam splitting made operational: Key features of the multi-turn extraction at the CERN Proton Synchrotron, Phys. Rev. Accel. Beams 20, 061001 (2017).

[13] S. Gilardoni, M. Giovannozzi, and C. Hernalsteens, First observations of intensity-dependent effects for transversely split beams during multiturn extraction studies at the CERN Proton Synchrotron, Phys. Rev. ST Accel. Beams 16, 051001 (2013).

[14] SHiP Collaboration, Report No. CERN-SPSC-2015-016; arXiv:1504.04956.

[15] A. Bazzani, G. Servizi, E. Todesco, and G. Turchetti, CERN Yellow Report No. 94-02, 1994.

[16] G. Franchetti and I. Hofmann, Particle trapping by nonlinear resonances and space charge, Nucl. Instrum. Methods Phys. Res., Sect. A 561, 195 (2006).

[17] I. Hofmann and G. Franchetti, Nonlinear phenomena in space-charge dominated beams, Nucl. Instrum. Methods Phys. Res., Sect. A 561, 137 (2006).

[18] G. Turchetti, A. Bazzani, F. Bergamini, S. Rambaldi, I. Hofmann, L. Bongini, and G. Franchetti, Frequency map analysis of resonances in a nonlinear lattice with space charge, Nucl. Instrum. Methods Phys. Res., Sect. A 464, 551 (2001).
[19] G. Franchetti, I. Hofmann, M. Giovannozzi, M. Martini, and E. Métral, Space charge and octupole driven resonance trapping observed at the CERN Proton Synchrotron, Phys. Rev. ST Accel. Beams 6, 124201 (2003).

[20] E. Métral, G. Franchetti, M. Giovannozzi, I. Hofmann, M. Martini, and R. Steerenberg, Observation of octupole driven resonance phenomena with space charge at the CERN Proton Synchrotron, Nucl. Instrum. Methods Phys. Res., Sect. A 561, 257 (2006).

[21] G. Franchetti, O. Chorniy, I. Hofmann, W. Bayer, F. Becker, P. Forck, T. Giacomini, M. Kirk, T. Mohite, C. Omet, A. Parfenova, and P. Schtt, Experiment on space charge driven nonlinear resonance crossing in an ion synchrotron, Phys. Rev. ST Accel. Beams 13, 114203 (2010).

[22] G. Franchetti, S. Gilardoni, A. Huschauer, F. Schmidt, and R. Wasef, Space charge effects on the third order coupled resonance, submitted for publication to Phys. Rev. Accel. Beams.

[23] L. Bongini, A. Bazzani, G. Turchetti, and I. Hofmann, Analysis of a model for resonant extraction of intense beams by normal forms and frequency map, Phys. Rev. ST Accel. Beams 4, 114201 (2001).

[24] C. Benedetti and G. Turchetti, An analytic map for space charge in a nonlinear lattice, Phys. Lett. A 340, 461 (2005).

[25] E. Todesco, M. Giovannozzi, and W. Scandale, Fast indicators of long-term stability, Part. Accel. 55, 27 (1996).

[26] M. Giovannozzi, W. Scandale, and E. Todesco, Dynamic aperture extrapolation in the presence of tune modulation, Phys. Rev. E 57, 3432 (1998).

[27] F. Kesting and G. Franchetti, Propagation of numerical noise in particle-in-cell tracking, Phys. Rev. ST Accel. Beams 18, 114201 (2015).

[28] E. Regenstreif, Report No. CERN 59-29, 1959.

[29] S. Gilardoni et al., in Tenth European Particle Accelerator Conference, edited by J. Poole and C. Petit-Jean-Genaz (Institute of Physics, London, United Kingdom, 2006), p. 264

[30] M. Juchno (private communication).

[31] R. Cappi, M. Giovannozzi, M. Martini, E. Métral, G. Métral, R. Steerenberg, and A.-S. Müller, in 2003 Particle Accelerator Conference, edited by J. Chew, P. Lucas, and S. Webber (IEEE Computer Society Press, Piscataway, 2003), p. 2913.

[32] S. Machida and M. Ikegami, Simulation of space charge effects in a synchotron, AIP Conf. Proc. 448, 73 (1998).

[33] J. D. Jackson, Classical Electrodynamics (Wiley, New York, 1975).

[34] K.-Y. Ng, Exact solutions for the longitudinal and transverse impedances of an off centered beam in a rectangular beam pipe, Part. Accel. 16, 63 (1984).

[35] Q. Shou, Q. Jiang, and Q. Guo, The closed-form solution for the 2D Poisson equation with a rectangular boundary, J. Phys. A 42, 205202 (2009). 